\title{
Resisting Three-Dimensional Manipulations in Distributed Wireless Spectrum Auctions
}

\author{
Dan Peng ${ }^{\dagger}$, Shuo Yang ${ }^{\dagger}$, Fan $\mathrm{Wu}^{\dagger}$, Guihai Chen ${ }^{\dagger}$, Shaojie Tang ${ }^{\S}$, and Tie Luo ${ }^{\natural}$ \\ ${ }^{\dagger}$ Shanghai Key Laboratory of Scalable Computing and Systems, Shanghai Jiao Tong University, China \\ ${ }^{\S}$ Department of Information Systems, University of Texas at Dallas, USA \\ ${ }^{\natural}$ Institute for Infocomm Research, A*STAR, Singapore \\ \{pd347,wnmmxy\}@sjtu.edu.cn; \{fwu, gchen\}@cs.sjtu.edu.cn; tangshaojie@gmail.com; luot@i2r.a-star.edu.sg
}

\begin{abstract}
Auctions are believed to be effective methods to solve the problem of wireless spectrum allocation. Existing spectrum auction mechanisms are all centralized and suffer from several critical drawbacks of the centralized systems, which motivates the design of distributed spectrum auction mechanisms. However, extending a centralized spectrum auction to a distributed one broadens the strategy space of agents from one dimension (bid) to three dimensions (bid, communication, and computation), and thus cannot be solved by traditional approaches from mechanism design. In this paper, we propose two distributed spectrum auction mechanisms, namely distributed VCG and FAITH. Distributed VCG implements the celebrated Vickrey-Clarke-Groves mechanism in a distributed fashion to achieve optimal social welfare, at the cost of exponential communication overhead. In contrast, FAITH achieves sub-optimal social welfare with tractable computation and communication overhead. We prove that both of the two proposed mechanisms achieve faithfulness, i.e., the agents' individual utilities are maximized, if they follow the intended strategies. We also implement FAITH and evaluate its performance in various setups. Evaluation results show that FAITH achieves superior performance compared with the Nash equilibrium based approach.
\end{abstract}

\section{INTRODUCTION}

The naturally limited radio spectrum is becoming more and more scarce with the increasing number of wireless applications. Unfortunately, traditional static spectrum allocations are expensive and inefficient, causing newly emerged wireless services and applications unable to meet their demands for spectrum [1]. To overcome the limitations of traditional spectrum allocation, auctions have become natural choices due to their fairness and efficiency.

In recent years, a number of spectrum auction mechanisms (e.g., [2]-[8]) have been proposed. These mechanisms achieve some attractive properties, such as strategy-proofness and approximate social welfare. Here, intuitively, strategy-proofness

This work was supported in part by the State Key Development Program for Basic Research of China (973 project 2014CB340303), in part by China NSF grant $61422208,61472252,61272443$ and 61133006 , in part by CCFIntel Young Faculty Researcher Program and CCF-Tencent Open Fund, in part by the Scientific Research Foundation for the Returned Overseas Chinese Scholars, State Education Ministry, and in part by Program for Changjiang Scholars and Innovative Research Team in University (IRT1158, PCSIRT) China. The opinions, findings, conclusions, and recommendations expressed in this paper are those of the authors and do not necessarily reflect the views of the funding agencies or the government. F. Wu is the corresponding author. means that one can maximize her payoff by truthfully revealing her private valuation on the spectrum; social welfare means the sum of auction winners' valuations on the allocated spectrum. However, these existing spectrum auction mechanisms rely on a centralized and trusted authority to perform as the auctioneer and to process the auction procedures.

The centralized spectrum auction mechanisms have several critical drawbacks [9]. The first is that the functionality of the centralized mechanisms is based on the assumption that there exists a trusted central authority. But in practice, especially in the secondary spectrum market for wireless networks, a trusted central authority may not always exist. The second drawback is that the scalability of the centralized spectrum auctions can be poor. Since the auctioneer needs to collect all the bids in order to calculate the auction outcome, the agents need reliable ways to deliver their bids to the auctioneer. Unfortunately, such communication channels may not always exist between the auctioneer and the agents in wireless networks, especially when the wireless network is not fully connected. The third drawback, which is not only limited to spectrum auction mechanisms, but also applies to centralized systems in general, is robustness. Once the central authority breaks down, the entire system collapses.

To tackle the above drawbacks of the centralized spectrum auction mechanisms, we propose to implement distributed spectrum auction mechanisms. However, designing a distributed spectrum auction mechanism is much more challenging.

Most of all, without the management of a central authority, the role of agents are now two-fold, i.e., not only to compete with each other for the wireless spectrum (as they do in centralized mechanisms), but also to cooperate in determining the outcome of the auction. This greatly broadens the strategy space of the agents from one dimension (i.e., bid reporting) to three dimensions (i.e., bid reporting, message passing, and computation) [10], and thus are beyond the scope of traditional mechanism design perspective.

Second, unlike conventional goods, wireless spectrum can be spatially reused by multiple agents as long as their transmissions do not reduce each other's Signal to Interference and Noise Ratio (SINR) below a predefined threshold. Such a unique property makes it computationally intractable when calculating an optimal spectrum allocation for a large scale 
wireless network, even in a centralized manner. Due to lack of global information of inter-agent interferences, optimizing the spectrum allocation with local knowledge in a distributed wireless network is really challenging.

Third, due to wireless devices' limited computation capability and communication bandwidth, traditional secure multiparty computation cannot be directly applied, given its high computation and communication overhead. Therefore, the problem of designing a manipulation-resistant distributed auction mechanism need to be carefully considered.

In this paper, we characterize the spectrum allocation problem from the perspective of distributed algorithmic mechanism design (DAMD) [9] and propose two complementary distributed auction mechanisms, i.e., distributed VCG and FAITH. Distributed VCG is an extension of the celebrated VickreyClarke-Groves (VCG) mechanism [11]-[13] to the distributed scenario. It collects bidding information bottom-up based on a carefully constructed pseudo-tree, and disseminates the optimal allocation top-down following the same tree structure. The payment for using the allocated spectrum is determined in the VCG manner. However, the optimal spectrum allocation is achieved at the cost of high communication overhead. Therefore, distributed VCG can only work in sparse secondary spectrum markets. We further present FAITH, which achieves sub-optimal spectrum allocation with bounded computation and communication overhead in general cases. Our analysis shows that both of the two proposed mechanisms are faithful, i.e., achieving Incentive Compatibility (IC), Communication Compatibility (CC), and Algorithm Compatibility (AC) [10], [14]. Here, intuitively, IC, $\mathrm{CC}$, and AC mean that none of the agents has the incentive to deviate from the prescribed information-revelation strategy, message-passing strategy, and computation strategy of the auction mechanism, respectively.

Our main contributions are listed as follows.

- To the best of our knowledge, we are the first to consider the problem of distributed algorithmic mechanism design for secondary wireless spectrum markets. We extend the celebrated VCG mechanism to a distributed scenario, and prove that our extension is a faithful implementation of spectrum auction mechanism, achieving optimal social welfare.

- Second, we propose a more practical and efficient faithful distributed spectrum auction mechanism, called FAITH. FAITH achieves sub-optimal social welfare with low computation and communication overhead.

- Finally, we implement FAITH and extensively evaluate its performance. Our evaluation results show that FAITH achieves good performance in terms of social welfare with low communication overhead.

The rest of the paper is organized as follows. In Section II, we present the technical preliminaries. Then, the distributed VCG and FAITH are presented in Section III and Section IV, respectively. In Section V, we evaluate FAITH and present numerical results. Then, we briefly review related work in Section VI. Finally, we conclude this paper in Section VII.

\section{PRELIMINARIES}

In this section, we describe our auction model for wireless spectrum allocation, and present related solution concepts.

\section{A. Model of Distributed Spectrum Auction}

We model the problem of channel allocation in the secondary spectrum market as a distributed auction, in which there are a number of orthogonal channels to be leased out and a set of channel buyers, called agents, who want to lease the channels to serve their subscribers and make profits. Multiple agents can share the same channel if they do not interfere with each other. Without the control of an auctioneer, a distributed auction is conducted by the rational agents themselves in the market. The objective of this auction is to efficiently select winners among the agents satisfying their interference constraints, and also to prevent the agents from manipulating the auction outcome.

Specifically, we consider a set $\mathbb{C}=\left\{c_{1}, c_{2}, \ldots, c_{m}\right\}$ of orthogonal and homogeneous channels. Information of the channels is public and known to the agents. Each channel can be simultaneously allocated to multiple non-conflicting agents, i.e., they can provide services to their subscribers simultaneously with an adequate SINR. As shown in papers [7], [15], the interference between the agents can be represented by a conflict graph. We assume that the agents in one auction belong to the same connected component in the conflict graph. For a conflict graph with multiple connected components, each connected component can conduct an independent distributed spectrum auction. We also assume that conflicting agents can communicate with each other through a commonly known control channel, i.e., the communication range of the agents on the control channel is larger than the interference range of them on working channels. This is backed by the existing communication protocols, e.g., the communication range of IEEE $802.11 \mathrm{~b}$ at a data rate of $1 \mathrm{Mbps}$ is normally larger than the interference range of IEEE $802.11 \mathrm{n}$ at $150 \mathrm{Mbps}$.

We also consider a set $\mathbb{A}=\left\{a_{1}, a_{2}, \ldots, a_{n}\right\}$ of agents. Each agent $a_{i} \in \mathbb{A}$ has a per-channel valuation $v_{i}$, which is commonly known as type in the literature and is private to the agent herself. The agent $a_{i}$ also has a strict demand of $d_{i}$ channels. Any winning agent $a_{i}$ has to pay $p_{i}$ for allocated channel(s). We define the utility of agent $a_{i}$ to be the difference between her total valuation and payment, i.e., $u_{i} \triangleq d_{i} \times v_{i}-p_{i}$. Similar to papers [16]-[18], we assume that there is a Credit Clearance Service (CCS), who neither participates in the auction to determine the allocation and payment, nor needs to be always online during the auction. In distributed VCG, the CCS collects the payments from the agents through an intermittently connected wireless overlay network. In FAITH, the CCS subtly controls agents' manipulated strategies on computation and communication by conducting an audit process.

In this paper, we consider that the agents are rational but helpful, meaning that although self-interested, each of the agents follows the prescriptions of the spectrum auction mechanism, if no unilateral deviation can lead to a better utility. We assume that there is no collusion among the agents. 
In contrast to the agents' individual objectives, the overall objective of the spectrum auction is to maximize social welfare $(S W)$, which is the sum of winning agents' valuations on their allocated channel(s), i.e.,

$$
S W \triangleq \sum_{a_{i} \in W}\left(d_{i} \times v_{i}\right),
$$

where $W$ is the set of winners.

\section{B. Solution Concepts}

Given the auction model, we review some important solution concepts used in this paper. First, we recall the definition of distributed mechanism.

Definition 1 (Distributed Mechanism [9], [14]). A distributed mechanism $\mathcal{M}=\left(g, \boldsymbol{\Sigma}, \boldsymbol{s}^{M}\right)$ defines a determination rule $g$, a feasible strategy space $\boldsymbol{\Sigma}=\Sigma_{1} \times \Sigma_{2} \times \ldots \times \Sigma_{n}$, and a prescribed strategy profile $s^{M}=\left(s_{1}^{M}, s_{2}^{M}, \ldots, s_{n}^{M}\right)$.

For any agent $a_{i}$, the intended strategy $s_{i}^{M}$ is composed of three sub-strategies, i.e., information-revelation strategy, message-passing strategy, and computation strategy.

Definition 2 (IC, CC, AC [10], [14]). A distributed mechanism achieves IC (resp. CC, AC) if no agent can gain higher utility by deviating from intended information-revelation strategy (resp. message-passing strategy and computation strategy) in an equilibrium.

Definition 3 (Ex-Post Nash Equilibrium [9], [10]). A strategy profile $s^{*}$ is an ex-post Nash equilibrium of a distributed mechanism $\mathcal{M}$, if for any agent $a_{i}$, any $s_{i}^{\prime} \neq s_{i}^{*}$, we have

$$
u_{i}\left(g\left(s_{i}^{*}, s_{-i}^{*}\right)\right) \geq u_{i}\left(g\left(s_{i}^{\prime}, s_{-i}^{*}\right)\right) .
$$

We now introduce the concept of faithful implementation.

Definition 4 (Faithful Implementation [10], [14]). A distributed mechanism $\mathcal{M}=\left(g, \boldsymbol{\Sigma}, s^{M}\right)$ is a faithful implementation of outcome $g\left(s^{M}\right)$ when the prescribed strategy profile $\boldsymbol{s}^{M}$ is an ex-post Nash equilibrium.

Intuitively, under a faithful distributed mechanism, the agents' individual utilities are maximized, if they follow the prescribed strategies.

\section{Distributed VCG}

In this section, we present a distributed extension of the celebrated VCG auction mechanism.

Definition 5 (VCG mechanism [11]-[13]). A mechanism $\left(f, p_{1}, \ldots, p_{n}\right)$ is Vickrey-Clarke-Groves (VCG) mechanism if

- Outcome function $f:\left(v_{1}, \ldots, v_{n}\right) \rightarrow \mathcal{K}$, ends up with $\boldsymbol{k}^{*}=$ $\operatorname{argmax}_{\boldsymbol{k} \in \mathcal{K}} \sum_{i} v_{i}(\boldsymbol{k})$, where $\mathcal{K}$ is a discrete choice set.

- Payment function $p_{i}\left(v_{1}, \ldots, v_{n}\right)=h_{i}\left(\boldsymbol{v}_{-i}\right)-\sum_{j \neq i} v_{j}\left(\boldsymbol{k}^{*}\right)$, where $h_{i}: V_{-i} \rightarrow \mathcal{R}$ (i.e., $h_{i}$ does not depend on $v_{i}$ ).

The outcome $\boldsymbol{k}^{*}$ in VCG mechanism achieves optimal social welfare. To implement the outcome function in a distributed manner, we propose a distributed channel allocation algorithm, which is inspired by the idea of Distributed Pseudo-tree (a) Conflict graph

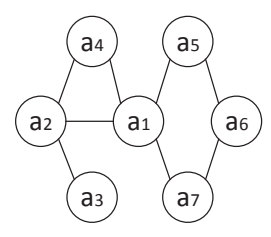

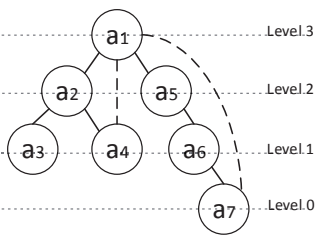

(b) Pseudo-tree
Fig. 1. Pseudo-tree construction

Optimization Procedure (DPOP) [19], but has clear differences from DPOP by supporting multi-channel requests and considering spectrum spatial reusability.

Our distributed VCG has three phases: pseudo-tree construction, channel assignment, and payment determination. It differs from the traditional VCG by (1) supporting parallel search for the optimal solution, and (2) imposing only polynomial computation overhead on the root agent in channel assignment.

\section{A. Pseudo-Tree Construction}

We construct a pseudo-tree from the conflict graph so that conflicting agents are organized in the same branch of the tree. The relative independence of nodes lying in different branches of the pseudo-tree facilitates parallel searches for the global optimal result [20], [21]. Fig. 1 shows an example of pseudotree construction, where Fig. 1(b) is a pseudo-tree constructed from the conflict graph shown in Fig. 1(a).

In the pseudo-tree, we define $P\left(a_{i}\right)$ and $C\left(a_{i}\right)$ be the set of parent and children of agent $a_{i}$, respectively. Here, $a_{i}$ 's parent and children are connected with $a_{i}$ by solid edges. We further define $P P\left(a_{i}\right)$ and $P C\left(a_{i}\right)$ as the set of pseudo parents and pseudo children of agent $a_{i}$, respectively. In contrast, an agent is connected with her pseudo parents and pseudo children by dashed edges. For example, in Fig. 1(b), $C\left(a_{1}\right)=\left\{a_{2}, a_{5}\right\}$, $P C\left(a_{1}\right)=\left\{a_{4}, a_{7}\right\}, P\left(a_{7}\right)=\left\{a_{6}\right\}, P P\left(a_{7}\right)=\left\{a_{1}\right\}$.

To construct the pseudo-tree, we can employ a distributed Depth-First Search (DFS) tree construction protocol [22]. Due to limitations of space, we omit the detailed algorithm. We assume that the pseudo-tree has already been constructed and every agent has known her parent, children, pseudo parents, pseudo children, and their levels in the pseudo-tree.

\section{B. Channel Assignment}

Our channel assignment algorithm consists of two phases: bottom-up social welfare aggregation and top-down channel choice propagation. It supports both single-channel demand and multi-channel demand. For clarity, we only discuss single-channel demand, and thus the selection domain is the same for any agent, i.e., $\forall a_{i} \in \mathbb{A}, d_{i}=1, D_{i}=$ $\left\{c_{1}, c_{2}, \ldots, c_{m}, N U L L\right\}$. We put $N U L L$ in agents' selection domains in order to let the agents choose nothing when they do not want to lease any channel.

Here, we define agent $a_{i}$ 's constraint view $C V\left(a_{i}\right)$ to be the set of $a_{i}$ 's parent and pseudo parents, and any other agents satisfying the following two conditions: (1) having higher level than $a_{i}$ and (2) having a pseudo child located in the subtree rooted at $a_{i}$ (e.g., $C V\left(a_{4}\right)=\left\{a_{1}, a_{2}\right\}$ and $\left.C V\left(a_{6}\right)=\left\{a_{1}, a_{5}\right\}\right)$. In our algorithm, “ $a_{i}: k_{i}$ ” means "when 
$a_{i}$ is allocated $k_{i}$ " and $v_{i}\left(a_{i}: k_{i}, a_{j}: k_{j}\right)$ is $a_{i}$ 's valuation over the channel allocation that $a_{i}$ is allocated $k_{i}$ and $a_{j}$ is allocated $k_{j}$, where $k_{i} \in D_{i}$ and $k_{j} \in D_{j}$. Note that $a_{i}$ 's valuation function equals to $a_{i}$ 's per channel valuation $v_{i}$ if and only if $a_{i}$ is allocated a channel and none of her neighbors are allocated the same channel.
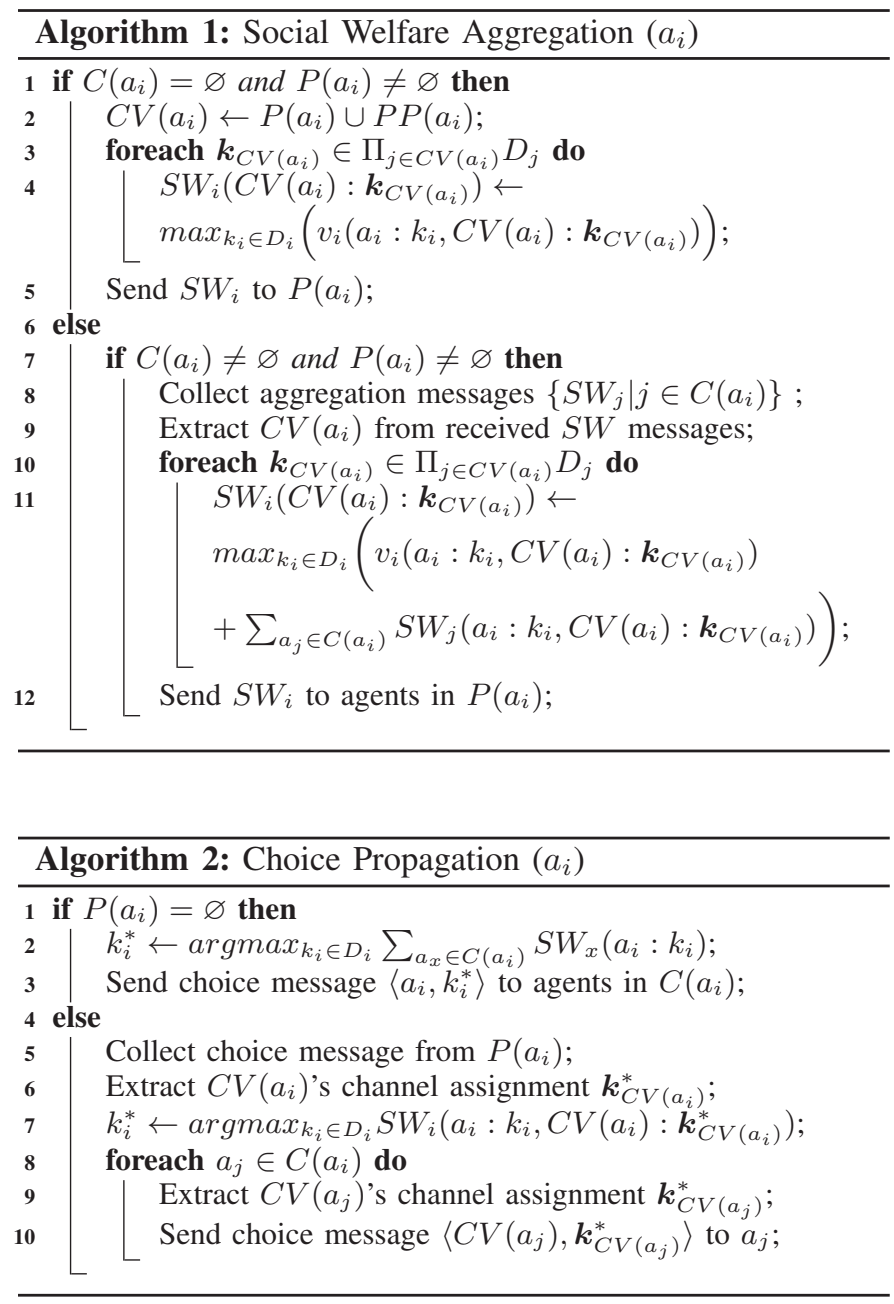

1) Social Welfare Aggregation: The bottom-up $S W$ aggregation, as shown in Algorithm 1, starts from leaf agents and goes up towards the root following the pseudo-tree edges. The $S W_{i}$ is the set of possible optimal social welfare that can be achieved by the subtree rooted at $a_{i}$, under each possible channel assignment of $C V\left(a_{i}\right)$. After collecting $S W$ messages from her children, an agent can compose her aggregation message and send it to her parent if she is not the root.

For a leaf agent $a_{i}$, if $P\left(a_{i}\right)=\left\{a_{j}\right\}$ and $P P\left(a_{i}\right)=\varnothing$, then $C V\left(a_{i}\right)=\left\{a_{j}\right\}$ and the social welfare that can be achieved at $a_{i}$ would only depend on her parent $a_{j}$. Thus the $S W_{i}$ sent from $a_{i}$ to $a_{j}$ would be a vector of the optimal social welfare that can be achieved at $a_{i}$, under each possible channel assignment of $a_{j}$. However, if $P P\left(a_{i}\right) \neq \varnothing$, then $C V\left(a_{i}\right)=$ $\left\{a_{j}\right\} \cup P P\left(a_{i}\right)$ and the social welfare that can be achieved at $a_{i}$, would depend on both her parent and pseudo parents. Thus, the $S W_{i}$ would be a hypercube of $1+\left|P P\left(a_{i}\right)\right|$ dimensions (one dimension for parent and the other $\left|P P\left(a_{i}\right)\right|$ for pseudo parents) of the tuple $\left\langle P\left(a_{i}\right), P P\left(a_{i}\right)\right\rangle$.

For an intermediate agent $a_{i}$, the social welfare that can be achieved by the subtree rooted at $a_{i}$ would be constrained by agents in her constraint view. After receiving all the $S W$ messages from her children, an intermediate agent can examine the $S W$ messages and get her children's constraint views and then extract her own constraint view $C V\left(a_{i}\right)$. After that, under each possible channel assignment of $C V\left(a_{i}\right)$, say $\boldsymbol{k}_{C V\left(a_{i}\right)}, a_{i}$ calculates the optimal social welfare that can be achieved by the subtree rooted at $a_{i}$, which is $S W_{i}\left(C V\left(a_{i}\right): \boldsymbol{k}_{C V\left(a_{i}\right)}\right)$.

2) Choice Propagation: The top-down choice propagation, as shown in Algorithm 2, starts from root agent and moves towards the leaves. After receiving all the $S W$ messages, the root agent calculates the overall social welfare under each of her own channel choice, then picks the optimal choice, and sends her choice message down to her children. For any nonroot agent $a_{i}$, based on the received choice message from her parent, $a_{i}$ picks her own channel choice $k_{i}^{*}$ that maximizes the social welfare for the subtree rooted at $a_{i}$, and sends the decision down to her children. The choice message received by $a_{i}$ from $P\left(a_{i}\right)$, contains not only her parent's choice, but also the choices of other agents in $C V\left(a_{i}\right)$.

When all the leaf agents have made their choices, the algorithm terminates. The channel assignment outcome $\boldsymbol{k}^{*}=$ $\left(k_{1}^{*}, k_{2}^{*}, \ldots, k_{n}^{*}\right)$, where $k_{i}^{*} \in D_{i}$, is the one that maximizes the overall social welfare.

\section{Payment Determination}

After determining the optimal channel assignment $\boldsymbol{k}^{*}$, we calculate the payment for each winner. We set $h_{i}\left(\boldsymbol{v}_{-i}\right)$ in VCG payment function to $\max _{\boldsymbol{k} \in \mathcal{K}} \sum_{j \neq i} v_{j}(\boldsymbol{k})$, then the payment of agent $a_{i}$ is

$$
p_{i}=\max _{\boldsymbol{k} \in \mathcal{K}} \sum_{j \neq i} v_{j}(\boldsymbol{k})-\sum_{j \neq i} v_{j}\left(\boldsymbol{k}^{*}\right) .
$$

We define $\boldsymbol{k}_{-i}^{*}=\operatorname{argmax}_{\boldsymbol{k} \in \mathcal{K}} \sum_{j \neq i} v_{j}(\boldsymbol{k})$, then

$$
p_{i}=\sum_{j \neq i} v_{j}\left(\boldsymbol{k}_{-i}^{*}\right)-\sum_{j \neq i} v_{j}\left(\boldsymbol{k}^{*}\right)=\sum_{j \neq i}\left(v_{j}\left(\boldsymbol{k}_{-i}^{*}\right)-v_{j}\left(\boldsymbol{k}^{*}\right)\right) \text {. }
$$

From the above payment scheme, we note that the payment for $a_{i}$ can be calculated without $a_{i}$. Thus, to calculate payment for $a_{i}$, we exclude $a_{i}$ from the conflict graph and create $\operatorname{DFS}\left(\mathbb{A}_{-i}\right)$ by modifying DFS $(\mathbb{A})$ : the highest descendant of $a_{i}$ that has a pseudo edge with an ancestor of $a_{i}$ turns the pseudo edge into a tree-edge. If such descendant does not exist, we exclude $a_{i}$ and her adjacent edges. For example, Fig. 2 shows the $\operatorname{DFS}\left(A_{-2}\right), \operatorname{DFS}\left(A_{-5}\right)$ and $\operatorname{DFS}\left(A_{-6}\right)$ after agent $a_{2}, a_{5}$ and $a_{6}$ are removed respectively from Fig. 1(b). Then, we run channel assignment algorithm on modified DFS $\left(\mathbb{A}_{-i}\right)$ to get $\boldsymbol{k}_{-i}^{*}$. If excluding $a_{i}$ causes more connected components, then we run channel assignment algorithm on each connected component once. Afterwards, each agent $a_{j} \neq a_{i}$ is asked to report $v_{j}\left(\boldsymbol{k}_{-i}^{*}\right)-v_{j}\left(\boldsymbol{k}^{*}\right)$ to the CCS, who then extracts payments from agents' accounts. We run this procedure for 


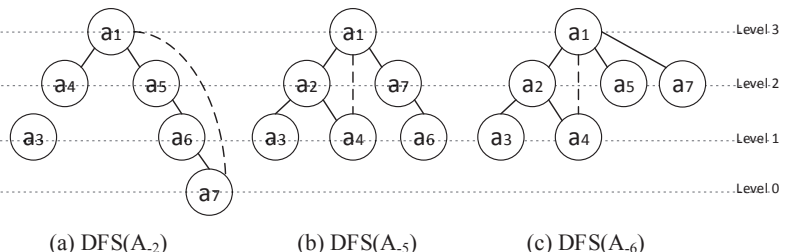

Fig. 2. $\operatorname{DFS}\left(A_{-i}\right)$

each $a_{i} \in W$, where $W$ is the set of winners, thus $|W|$ times, to calculate payments for all agents.

\section{Mechanism Analysis}

Theorem 1. Distributed VCG mechanism is a faithful implementation.

Proof. When agents follow the prescribed strategies $s^{*}=$ $\left(s_{1}^{*}, \ldots, s_{n}^{*}\right)$, the optimal allocation $\boldsymbol{k}^{*}$ can be achieved, then for any agent $a_{i}, a_{i}$ 's utility is

$$
\begin{aligned}
u_{i}\left(g\left(s_{i}^{*}, \boldsymbol{s}_{-i}^{*}\right)\right) & =v_{i}\left(\boldsymbol{k}^{*}\right)-p_{i} \\
& =v_{i}\left(\boldsymbol{k}^{*}\right)-\sum_{j \neq i} v_{j}\left(\boldsymbol{k}_{-i}^{*}\right)+\sum_{j \neq i} v_{j}\left(\boldsymbol{k}^{*}\right) \\
& =\sum_{j \in \mathbb{A}} v_{j}\left(\boldsymbol{k}^{*}\right)-\sum_{j \neq i} v_{j}\left(\boldsymbol{k}_{-i}^{*}\right) .
\end{aligned}
$$

If agent $a_{i}$ personally chose to deviate from $s_{i}^{*}$ to $s_{i}^{\prime} \neq s_{i}^{*}$, then the channel assignment outcome may change to $\boldsymbol{k}^{\prime}$. Since $\boldsymbol{k}^{*}$ maximizes social welfare, then $\sum_{j \in \mathbb{A}} v_{j}\left(\boldsymbol{k}^{\prime}\right) \leq$ $\sum_{j \in \mathbb{A}} v_{j}\left(\boldsymbol{k}^{*}\right)$. As $a_{i}$ 's payment would not be influenced by her manipulation, $a_{i}$ 's utility under this situation is

$$
\begin{aligned}
u_{i}\left(g\left(s_{i}^{\prime}, \boldsymbol{s}_{-i}^{*}\right)\right) & =\sum_{j \in \mathbb{A}} v_{j}\left(\boldsymbol{k}^{\prime}\right)-\sum_{j \neq i} v_{j}\left(\boldsymbol{k}_{-i}^{*}\right) \\
& \leq \sum_{j \in \mathbb{A}} v_{j}\left(\boldsymbol{k}^{*}\right)-\sum_{j \neq i} v_{j}\left(\boldsymbol{k}_{-i}^{*}\right)=u_{i}\left(g\left(s_{i}^{*}, \boldsymbol{s}_{-i}^{*}\right)\right)
\end{aligned}
$$

which means that under the prescribed strategy profile, following the prescribed strategy maximizes one's utility. Thus the strategy profile is an ex-post Nash equilibrium and the distributed VCG is a faithful distributed mechanism.

The number of messages that distributed VCG produces is polynomial. However, the size of message for every agent $a_{i}$, except the root agent, is exponential to the number of agents in her constraint view, $\left|C V\left(a_{i}\right)\right|$. It is because that the agent has to calculate optimal welfare for the subtree, under each possible channel assignment of $C V\left(a_{i}\right)$. We note that the root agent, only burdens an overhead which is polynomial to the total number of channels, by choosing the channel which maximizes the overall social welfare.

\section{FAITH}

In this section, we propose a more practical distributed spectrum auction, FAITH, to incentivize the rational agents towards an efficient spectrum allocation in ex-post Nash equilibrium. FAITH overcomes the computation and communication intractability of the distributed VCG spectrum auction, and thus can be extended to large scale spectrum markets.

\section{A. Design Rationale}

In most of the truthful centralized spectrum auctions, an auctioneer sorts the agents in a non-increasing order of bids, greedily allocates channels to agents without violating the conflict constraints, and charges each winning agent with the critical price [23]. Let $\mathbb{N}_{i}$ denote an agent $a_{i}$ 's set of conflicting agents, called neighbors. After exchanging bids with neighbors, $a_{i}$ can divide $\mathbb{N}_{i}$ into preemptive neighbor set $\mathbb{P N}_{i}=\left\{a_{j} \mid a_{j} \succ a_{i}, a_{j} \in \mathbb{N}_{i}\right\}$ and feedback neighbor set $\mathbb{F} \mathbb{N}_{i}=\left\{a_{j} \mid a_{i} \succ a_{j}, a_{j} \in \mathbb{N}_{i}\right\}$, where $\succ$ defines a priority order (i.e., $a_{i} \succ a_{j}$, if $b_{i}>b_{j}$, or $b_{i}=b_{j}$ and $i<j$ ).

We observe that an agent's channel allocation is only affected by her preemptive neighbors, and her allocation will directly influence her feedback neighbors' selections. This observation indicates that propagating and gathering information within local neighborhoods in a well designed order is enough to determine the channel allocation. This local effect makes the distributed implementation of the centralized auction possible.

However, simply letting the agents themselves handle the auction process may lead to manipulation of computation and communication. Therefore, besides incentive compatibility achieved by traditional centralized auctions, a distributed auction should also resist the computation and communication manipulations. We observe that in a distributed spectrum auction, the computation and communication of an agent is responded and confirmed by at least one of her neighbors, i.e., every agent acts both as a principal for herself, and as a witness for all of her neighbors. Exploiting agents' dual roles can provide necessary information for the CCS to verify agents' behaviors and to enable a "catch and punish" scheme (i.e., check the consistency of the information and penalize a deviation with a fine heavier than what one can gain).

\section{B. Design Details}

FAITH has two phases: (1) Bid Exchange and (2) Channel Selection and Payment Calculation. Agents carry out the two phases autonomously and independently without the participation of any centralized party.

1) Bid Exchange: In this phase, the agents exchange bid statement messages (MSGBs) with neighbors to get local bidding information. Each agent $a_{i} \in \mathbb{A}$ sends her bid statement message, which is formatted as

$$
\operatorname{MSGB}_{i}=<\mathrm{BID}, i, b_{i}, d_{i}>,
$$

to all of her neighbors $\mathbb{N}_{i}$. Upon receiving a bid statement message $\mathrm{MSGB}_{j}$ from a neighbor $a_{j}$, agent $a_{i}$ adds agent $a_{j}$ into her preemptive neighbor set $\mathbb{P N}_{i}$, if $a_{j} \succ a_{i}$; otherwise, $a_{i}$ adds $a_{j}$ into her feedback neighbor set $\mathbb{F} \mathbb{N}_{i}$. After the bid exchange phase, each of the agents gets her preemptive neighbor set and feedback neighbor set.

2) Channel Selection and Payment Calculation: Although logically separated, the processes of channel selection and payment calculation can be integrated together in order to reduce the number of messages involved in the distributed spectrum auction mechanism. The pseudo-code of this integrated process is shown in Algorithm 3. 


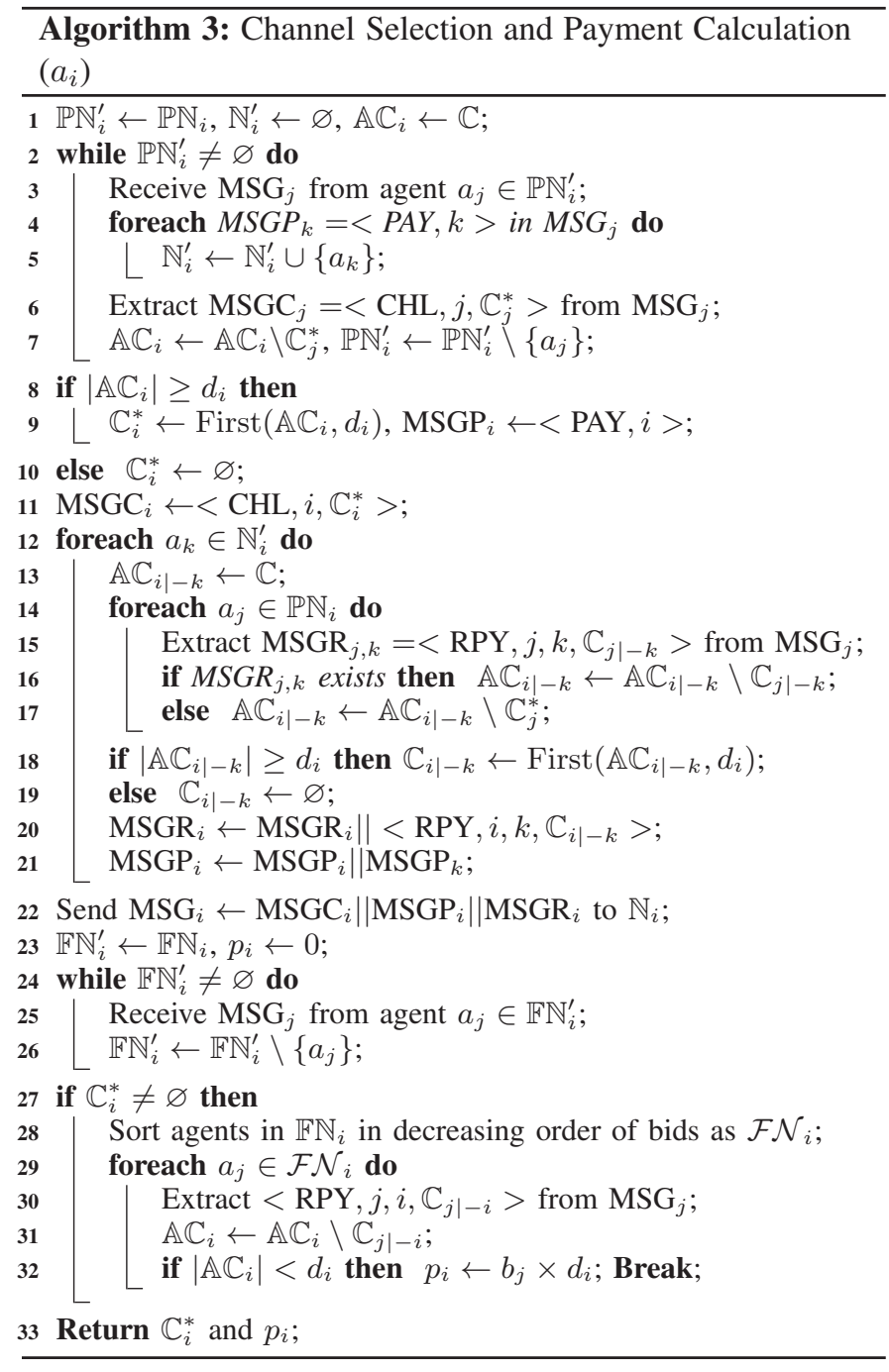

We start from describing the distributed channel selection algorithm based on the locally collected bidding information, and then specify how to combine information needed for payment calculation.

In the process of channel selection, each agent $a_{i}$ uses channel selection message (MSGC) to inform neighbors of her selected channel set $\mathbb{C}_{i}^{*}$, in the format as

$$
\mathrm{MSGC}_{i}=<\mathrm{CHL}, i, \mathbb{C}_{i}^{*}>\text {. }
$$

As discussed in Section IV-A, the channel selection of one agent is only affected by the selection of her preemptive neighbors. Thus, agent $a_{i}$ first collects MSGCs from her preemptive neighbors in $\mathbb{P N}_{i}$, and updates her available channel set $\mathbb{A C}_{i}$ by deactivating the channels that are already selected by her preemptive neighbors (Lines 2 to 7). Then, if there are enough channels left, she selects the first $d_{i}$ channel(s) from $\mathbb{A} \mathbb{C}_{i}$ as her own selected channel set

$$
\mathbb{C}_{i}^{*} \leftarrow \operatorname{First}\left(\mathbb{A C}_{i}, d_{i}\right)
$$

If $\mathbb{C}_{i}^{*} \neq \varnothing$, then $a_{i}$ is a winning agent (Lines 8 to 11 ).
The next step is to calculate each winning agent's payment. We employ the critical price as winning agent $a_{i}$ 's payment, i.e., the minimum price for $a_{i}$ to win in the spectrum auction. While an agent $a_{i}$ 's channel selection is affected by her preemptive neighbors, her payment is determined by her feedback neighbors. Consequently, to calculate $a_{i}$ 's payment, we need to ask $a_{i}$ 's feedback neighbors to provide necessary information, which is their channel selection if the agent $a_{i}$ does not participate in the spectrum auction. Each winning agent $a_{i}$ sends a payment determination request message

$$
\operatorname{MSGP}_{i}=<\text { PAY, } i>
$$

to her feedback neighbors (Line 9). Since $a_{i}$ 's feedback neighbors' channel selection can be affected by the agents that do not have direct connections with $a_{i}$, the payment determination request message $\mathrm{MSGP}_{i}$ needs to be further forwarded by the agents (Line 21). However, the total number of forwarding is bounded by the number of agents.

Upon receiving a payment determination request message $\operatorname{MSGP}_{k}$, agent $a_{i}$ first checks whether there are sufficient channels left, given her preemptive neighbors' selection if agent $a_{k}$ does not participate in the spectrum auction, i.e.,

$$
\mathbb{A} \mathbb{C}_{i \mid-k}=\mathbb{C}-\bigcup_{j \in \mathbb{P N}_{i}} \mathbb{C}_{j \mid-k},
$$

where $\mathbb{C}_{j \mid-k}$ denotes agent $a_{j}$ 's channel selection if agent $a_{k}$ is absent from the auction (Lines 14 to 17). If $\left|\mathbb{A C}_{i \mid-k}\right| \geq d_{i}$, agent $a_{i}$ sets $\mathbb{C}_{i \mid-k} \leftarrow \operatorname{First}\left(\mathbb{A C}_{i \mid-k}, d_{i}\right)$; otherwise, $\mathbb{C}_{i \mid-k} \leftarrow$ $\varnothing$ (Line 18 and 19). The agent $a_{i}$ encapsulates this selection into $\mathrm{MSGR}_{i}$ (Line 20), i.e.,

$$
\operatorname{MSGR}_{i} \leftarrow \operatorname{MSGR}_{i} \|<\mathrm{RPY}, i, k, \mathbb{C}_{i \mid-k}>
$$

Noting that sending the three different kinds of messages (i.e., $\mathrm{MSGC}_{i}, \mathrm{MSGP}_{i}$, and $\mathrm{MSGR}_{i}$ ) separately may introduce extra overhead for MAC layer coordination, we combine all of these three kinds of messages together

$$
\mathrm{MSG}_{i} \leftarrow \mathrm{MSGC}_{i}\left\|\mathrm{MSGP}_{i}\right\| \mathrm{MSGR}_{i},
$$

and utilize the broadcast of the wireless communication media to send the integrated messages in a single shot (Line 22).

After collecting replies from all her feedback neighbors (Line 24 to 26), agent $a_{i}$ can calculate her payment, if she is a winning agent. Here, she sorts her feedback neighbors in a decreasing order of bids as $\mathcal{F} \mathcal{N}_{i}$ (Line 28), and then follows the order to determine her critical price $b_{j}$, if it exists (Lines 29 to 32). The payment is $p_{i} \leftarrow b_{j} \times d_{i}$ (Line 32).

Toy Example: Fig. 3 shows a toy example for channel selection and payment calculation. We consider four agents $\mathbb{A}=\left\{a_{1}, a_{2}, a_{3}, a_{4}\right\}$, and 2 channels $\mathbb{C}=\left\{c_{1}, c_{2}\right\}$ for allocation. For clarity, we assume that each of the agents demands a single channel.

Each agent keeps a local ranking of agents (e.g., $a_{1}$ gets $a_{3} \succ a_{1} \succ a_{4}$, and $a_{3}$ gets $a_{3} \succ a_{2} \succ a_{1}>a_{4}$ ) after the bid exchange phase. Based on the ranking, agent sequentially selects one channel within her neighborhood. For agent $a_{3}$, she 


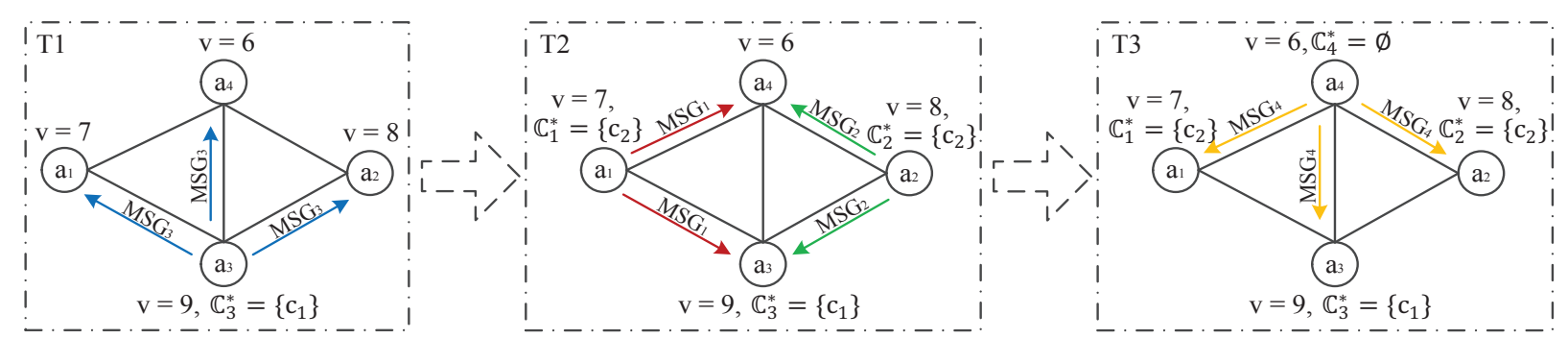

Fig. 3. Message flow for channel selection and payment calculation with 4 agents and 2 channels.

\begin{tabular}{|c|c|c|c|c|}
\hline Msg Agent & $a_{1}$ & $a_{2}$ & $a_{3}$ & $a_{4}$ \\
\hline MSGB & $<\mathrm{BID}, 1,7,1>$ & $<\mathrm{BID}, 2,8,1>$ & $<\mathrm{BID}, 3,9,1>$ & $<\mathrm{BID}, 4,6,1>$ \\
\hline Ranking & $a_{3} \succ a_{1} \succ a_{4}$ & $a_{3} \succ a_{2} \succ a_{4}$ & $a_{3} \succ a_{2} \succ a_{1} \succ a_{4}$ & $a_{3} \succ a_{2} \succ a_{1} \succ a_{4}$ \\
\hline MSGC & $<\mathrm{CHL}, 1,\left\{c_{2}\right\}>$ & $<$ CHL $, 2,\left\{c_{2}\right\}>$ & $<\mathrm{CHL}, 3,\left\{c_{1}\right\}>$ & $<\mathrm{CHL}, 4, \varnothing>$ \\
\hline MSGP & $<\mathrm{PAY}, 3><\mathrm{PAY}, 1>$ & $<\mathrm{PAY}, 3><\mathrm{PAY}, 2\rangle$ & $<\mathrm{PAY}, 3>$ & $<\mathrm{PAY}, 3><\mathrm{PAY}, 1><\mathrm{PAY}, 2>$ \\
\hline MSGR & $<\mathrm{RPY}, 1,3,\left\{c_{1}\right\}>$ & $<\mathrm{RPY}, 2,3,\left\{c_{1}\right\}>$ & & $\begin{array}{c}<\mathrm{RPY}, 4,3,\left\{c_{2}\right\}><\mathrm{RPY}, 4,2, \varnothing> \\
<\mathrm{RPY}, 4,1, \varnothing>\end{array}$ \\
\hline
\end{tabular}

MESSAGES TRANSMITTED IN THE NETWORK.

does not need to consider any preemptive selections, since she ranks the highest in her neighborhood. So she selects a channel $c_{1}$, broadcasts her message $\mathrm{MSG}_{3}=\mathrm{MSGC}_{3}|| \mathrm{MSGP}_{3}$, and waits for feedback neighbors' replies to calculate her payment. Upon receiving $\mathrm{MSG}_{3}$, agents $a_{1}$ and $a_{2}$ can run Algorithm 3 concurrently, since they are out of conflict. Agent $a_{1}$ updates her available channel set, selects a channel $c_{2}$, selects a payment determining channel $c_{1}$ assuming that $a_{3}$ is absent, and broadcasts her message $\mathrm{MSG}_{1}=\mathrm{MSGC}_{1}|| \mathrm{MSGP}_{1}|| \mathrm{MSGR}_{1}$. Agent $a_{2}$ runs the same process. Finally, agent $a_{4}$ collects messages from all her preemptive neighbors and responds her own message $\mathrm{MSG}_{4}$. Thereafter, winning agents extract feedback messages to find critical price and calculate payment (i.e., $p_{1}=0, p_{2}=0, p_{3}=6$ ). Table I lists the content of corresponding messages.

3) Consistency Check: To guarantee faithfulness, the consistency of the communication and computation should be checked. Note that each message sent in the spectrum auction has at least two copies (i.e., one at the sender and the other at the receiver) in the network. We require each agent to submit the messages she sent and received to the CCS, when clearing a transaction. After collecting all the messages, the CCS can check the messages, authorize the channel allocation and collect the payment. If a mismatch is detected, the involved agents have to pay a penalty which is higher than the largest possible utility one can gain by cheating. The CCS does not always need to have a reliable communication channel with each agent, or participate in the spectrum auction process. The CCS just needs to check the consistency and clear the transaction when a connection is available.

\section{Mechanism Analysis}

In this subsection, we show that FAITH meets the design requirements for distributed mechanism, especially in terms of network complexity and faithfulness.

1) Network Complexity: Feigenbaum et al. [9] proposed the concept of network complexity with respect to five metrics to measure the complexity of a distributed algorithm executed over an interconnected network $\mathbb{G}=(\mathbb{V}, \mathbb{E})$, where $\mathbb{V}=\mathbb{A}$ and $\mathbb{E}$ contains all the communication links among the agents in $\mathbb{A}$. Here we demonstrate the network complexity of FAITH in the following five metrics.

- Total number of messages sent over $\mathbb{G}$ : Every agent broadcasts two messages - one for bid exchange, and the other for integrated channel selection and payment calculation resulting in $4|\mathbb{E}|$ messages.

- Maximum number of messages sent over any link in $\mathbb{G}$ : There are 4 messages on each link due to mutual message sending in the two phases.

- Maximum size of a message: In the worst case, the agent with the lowest bid may inherit all the payment determination request messages from her preemptive neighbors (i.e., the agent that ranks lowest in a ring topology will extract all other agents' payment determination request messages when there are more than one channels being auctioned), which will result in a merged MSG with $2|\mathbb{V}|$ sub-messages (i.e., 1 for MSGC, $|\mathbb{V}|$ for MSGP, and $|\mathbb{V}|-1$ for MSGR). Since maximum length of each sub-message is a constant $c$-byte, the maximum size of a message is $2 c|\mathbb{V}|$.

- Local computation overhead: The toughest part throughout the mechanism is the payment determining channel reselection, which takes $O(\delta|\mathbb{V}|)$ time in the worst case, where $\delta$ is the maximum degree of the network.

- Storage overhead at each node: Every agent is required at most $O(\delta|\mathbb{V}|)$ space to store propagated messages and local outcome in the worst case.

2) Faithfulness: Given a centralized mechanism which is truthful, we can prove that a distributed mechanism is faithful by combining the other two properties: strong communication compatibility (strong-CC) and strong algorithm compatibility (strong-AC) [10]. Here the term of "strong" demonstrates that the three aspects of compatibility are independent, that is, no agent can get higher utility by deviating from the intended information-revelation/message-passing/computation strategy, whatever her other two actions are, when other agents follow the intended strategies. We assume that, for each agent, 
a complete implementation of the auction is much preferable than dropping out without any affirmed outcome.

In FAITH, the intended strategy for each agent is to report bidding information truthfully, pass messages correctly, and compute channel selection, reselection and payment correctly. A rational agent $a_{i}$ may deviate from the following aspects to increase her utility:

- Misreport: Report false bidding information $\left\langle b_{i}, d_{i}\right\rangle$.

- Miscommunication: Drop or change neighbors' messages, i.e., $\mathrm{MSGB}_{j}$ and $\mathrm{MSG}_{j}$, or withhold her own messages.

- Miscalculation: Divide neighbors into wrong sets, determine channel (re)selection $\mathbb{C}_{i}^{*}, \mathbb{C}_{i \mid-k}^{*}$ or payment $p_{i}$ incorrectly.

Theorem 2. FAITH is a faithful distributed implementation of the critical price-based spectrum allocation mechanism.

Proof. (Sketch) To prove this theorem, we show that FAITH satisfies centralized truthfulness, strong-CC and strong-AC.

- The corresponding centralized spectrum auction is demonstrated to be truthful in [7].

- FAITH satisfies strong-CC. Based on the redundant messages and "catch and punish" scheme, the CCS can detect communication deviation. Agent $a_{i}$ will not drop or change neighbor $a_{j}$ 's messages, since they are originally generated and kept by agent $a_{j}$, and any mismatch will be caught and punished in consistency check. Agent $a_{i}$ has no incentive to withhold her messages and block the auction. Thus, agent $a_{i}$ would follow the intended message-passing strategy.

- FAITH satisfies strong-AC. Agent $a_{i}$ divides her neighbors into two sets with different priorities. A unilateral miscalculation will breach determination order and cause communication chaos. Also, miscalculation can be detected by the CCS in consistency check. For example, in Fig. 3, based on $\mathrm{MSGB}_{1}$ and $\mathrm{MSGB}_{3}$, both $a_{1}$ and $a_{3}$ should rank $a_{3} \succ a_{1}$. Through consistency check, the CCS can guide the calculation to be correctly done. Agent $a_{i}$ selects (reselects) channel sets based on her preemptive neighbors' selections $\mathbb{C}_{j}\left(\mathbb{C}_{j \mid-k}\right)$, and calculates payment based on her feedback neighbors' selections $\mathbb{C}_{j \mid-i}$. All the necessary information is contained in $\mathrm{MSG}_{j}$ and sent to the CCS. Since any cheating will be caught and punished, agent $a_{i}$ has no incentive to deviate from the intended computation strategy.

According to the specification in [10], FAITH is a faithful distributed implementation of the critical price-based spectrum allocation mechanism.

\section{NUMERICAL RESUltS}

In this section, we evaluate the performance of FAITH on allocation efficiency and transmission overhead. Although the distributed VCG can achieve optimal social welfare, it is impractical in large scale scenarios, and thus we do not evaluate its performance here. We generate agents randomly in a square area of $2500 \mathrm{~m} \times 2500 \mathrm{~m}$, and apply a distance-based interference model [7], [15] to form the conflict graph. In our setting, any two agents within $250 \mathrm{~m}$ will conflict with each other and thus cannot use the same channel simultaneously.

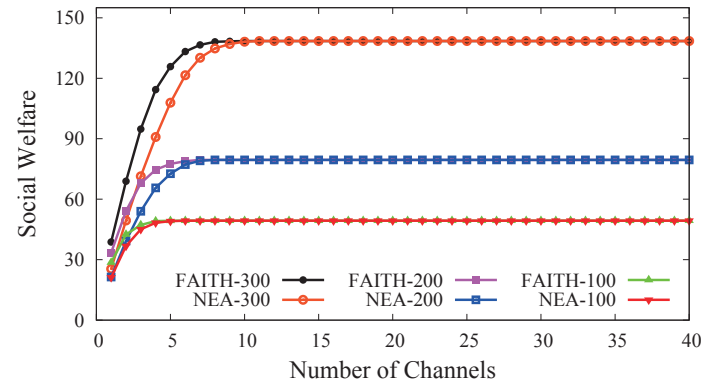

(a) Single-Channel Demand

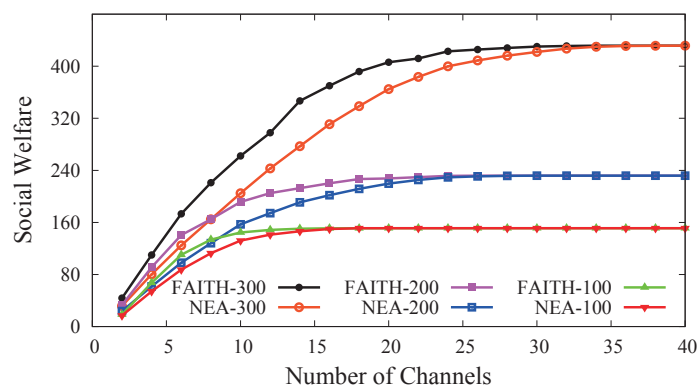

(b) Multi-channel Demand

Fig. 4. Social Welfare of FAITH $v s$. NEA.

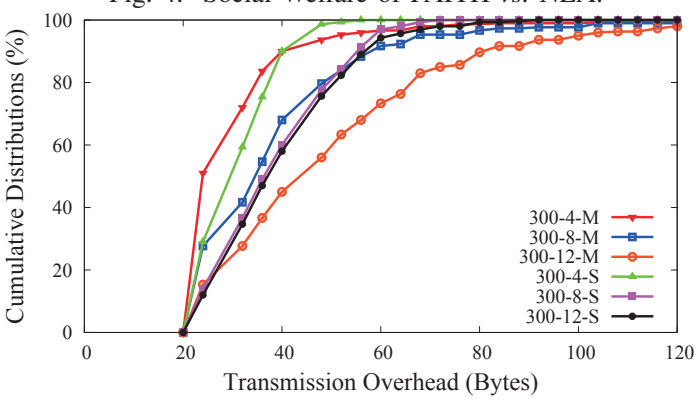

(a) Varying number of channels

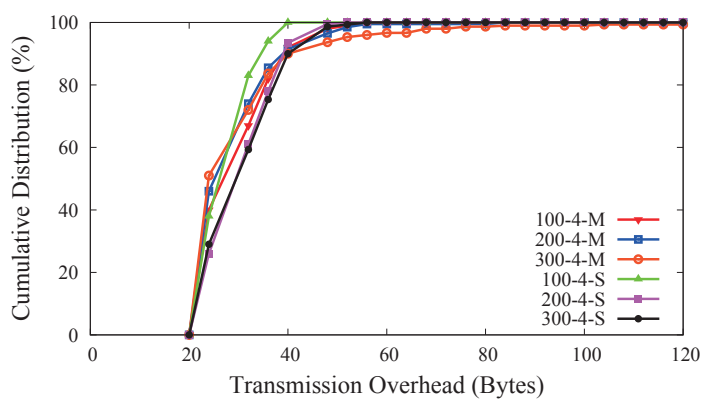

(b) Varying number of agents

Fig. 5. Transmission Overhead of FAITH.

The number of agents ranges from 100 to 300, and the number of channels ranges from 1 to 40. Without loss of generality, we uniformly distribute the bids of agents in $(0,1]$, and the channel demands in $\{1,2,3,4,5\}$. The results are averaged over 1000 runs.

\section{A. Allocation Efficiency}

We evaluate the allocation efficiency of FAITH in terms of social welfare, and compare it with a distributed Nash equilibrium based channel allocation algorithm [24] (denoted 
by NEA in our evaluation). Fig. 4 shows the results.

We observe that social welfare of both algorithms grows with the number of channels and finally reaches saturation, where every agent meets her demand. Besides, FAITH outperforms NEA with much higher social welfare before the saturation, since FAITH considers agents'priorities in the allocation, while NEA allows the agents to compete for channels in an arbitrary way. Meanwhile, due to the intensified competition, there is a lag of saturation in multi-channel demand when compared with single-channel demand (e.g., FAITH saturates at 8 channels for 300 agents with single-channel demand, while 30 channels are required for multi-channel demand).

\section{B. Transmission Overhead}

We also measure FAITH's per-agent transmission overhead, which is defined as the total size of messages each agent generates. Fig. 5 shows the cumulative distribution of transmission overhead in bytes, where " $\mathrm{n}-\mathrm{m}-\mathrm{S} / \mathrm{M}$ " denotes that $\mathrm{n}$ agents bid for $\mathrm{m}$ channels with single(S)/multi(M)-channel demand.

According to Fig. 5(a), we observe that the transmission overhead increases along with the number of channels until the allocation saturates (e.g., about $90 \%$ of agents transmit no more than 40 bytes in "300-4-M", while the percentage is only $67 \%$ in " $300-8-\mathrm{M}$ ", and $45 \%$ in "300-12-M"). This is because that more channels will result in more winners and thus more messages are generated for channel reselection and payment determination. However, the transmission overhead remains stable after saturation (e.g., the coincident distribution for "300-8-S" and "300-12-S"). Fig. 5(b) present the transmission overhead with varying number of agents. The similar cumulative distribution indicates that FAITH is scalable in distributed scenarios.

\section{RELATED WORK}

Various spectrum auction mechanisms have been proposed to improve spectrum utilization and allocation fairness over rational agents. VERITAS [7] and SMALL [6] are singlesided truthful auctions. TRUST [8] considers double auction. Al-Ayyoub et al. [2] proposed a truthful spectrum auction with guaranteed expected revenue. Dong et al. [3] studied combinatorial auction. TAHES [4] addresses heterogeneous spectrum in a double auction. SPRING [5] is strategy-proof and privacy preserving. All of these mechanisms are centralized. In contrast, we consider distributed mechanism design.

Feigenbaum et al. [25] initiated the study of distributed algorithmic mechanism design (DAMD) on multicast transmission, and articulated the concept of network complexity to measure the computation efficiency for distributed models. Later, the agenda was extended to lowest-cost interdomain routing [26]. Shneidman and Parks [10], [14] studied players' strategy space in distributed scenarios, introduced the notions of communication compatibility and algorithm compatibility, and proposed general principles to guide distributed mechanism design. Yet, no studies have been proposed for wireless spectrum allocation, which requires further consideration about the spatial reusability of spectrum.

\section{CONCLUSION}

In this paper, we have modeled the problem of wireless spectrum allocation as a distributed auction, and have proposed two faithful distributed auction mechanisms, namely distributed VCG and FAITH. We have implemented FAITH, and have analyzed its economic property and network complexity. Compared with an existing Nash equilibrium based approach, FAITH can achieve higher social welfare with low communication overhead.

\section{REFERENCES}

[1] R. Berry, M. L. Honig, and R. Vohra, "Spectrum markets: motivation, challenges, and implications," IEEE Communications Magazine, vol. 48, no. 11 , pp. $146-155,2010$.

[2] M. Al-Ayyoub and H. Gupta, "Truthful spectrum auctions with approximate revenue," in IEEE INFOCOM 2011.

[3] M. Dong, G. Sun, X. Wang, and Q. Zhang, "Combinatorial auction with time-frequency flexibility in cognitive radio networks," in IEEE INFOCOM 2012.

[4] X. Feng, Y. Chen, J. Zhang, Q. Zhang, and B. Li, "TAHES: Truthful double auction for heterogeneous spectrums," in IEEE INFOCOM 2012.

[5] Q. Huang, Y. Tao, and F. Wu, "Spring: A strategy-proof and privacy preserving spectrum auction mechanism," in IEEE INFOCOM 2013.

[6] F. Wu and N. Vaidya, "SMALL: A strategy-proof mechanism for radio spectrum allocation," in IEEE INFOCOM 2011.

[7] X. Zhou, S. Gandhi, S. Suri, and H. Zheng, "eBay in the sky: strategyproof wireless spectrum auctions," in ACM MobiCom 2008

[8] X. Zhou and H. Zheng, "TRUST: A general framework for truthful double spectrum auctions," in IEEE INFOCOM 2009.

[9] J. Feigenbaum, M. Schapira, and S. Shenker, Distributed algorithmic mechanism design. Cambridge University Press, 2007, pp. 363-384.

[10] J. Shneidman and D. C. Parkes, "Specification faithfulness in networks with rational nodes," in ACM PODC 2004.

[11] E. H. Clarke, "Multipart pricing of public goods," Public choice, vol. 11, no. 1, pp. 17-33, 1971.

[12] T. Groves, "Incentives in teams," Econometrica: Journal of the Econometric Society, pp. 617-631, 1973.

[13] W. Vickrey, "Counterspeculation, auctions, and competitive sealed tenders," The Journal of Finance, vol. 16, no. 1, pp. 8-37, 1961.

[14] D. C. Parkes and J. Shneidman, "Distributed implementations of vickreyclarke-groves mechanisms," in AAMAS 2004.

[15] K. Jain, J. Padhye, V. N. Padmanabhan, and L. Qiu, "Impact of interference on multi-hop wireless network performance," in ACM MobiCom 2003.

[16] W. Wang, S. Eidenbenz, Y. Wang, and X.-Y. Li, "OURS: optimal unicast routing systems in non-cooperative wireless networks," in $A C M$ MobiCom 2006.

[17] S. Zhong, J. Chen, and Y. R. Yang, "Sprite: A simple, cheat-proof, credit-based system for mobile ad-hoc networks," in IEEE INFOCOM 2003.

[18] S. Zhong and F. Wu, "On designing collusion-resistant routing schemes for non-cooperative wireless ad hoc networks," in ACM MobiCom 2007.

[19] A. Petcu and B. Faltings, "A scalable method for multiagent constraint optimization," in IJCAI 2005.

[20] R. Dechter, Constraint processing. Morgan Kaufmann, 2003.

[21] E. C. Freuder, "A sufficient condition for backtrack-bounded search," Journal of the ACM (JACM), vol. 32, no. 4, pp. 755-761, 1985.

[22] S. Makki and G. Havas, "Distributed algorithms for constructing a depthfirst-search tree," in IEEE ICPP 1994.

[23] R. B. Myerson, "Optimal auction design," Mathematics of operations research, vol. 6, no. 1, pp. 58-73, 1981.

[24] M. Felegyhazi, M. Cagalj, S. S. Bidokhti, and J.-P. Hubaux, "Noncooperative multi-radio channel allocation in wireless networks," in IEEE INFOCOM 2007

[25] J. Feigenbaum, C. H. Papadimitriou, and S. Shenker, "Sharing the cost of multicast transmissions1," Journal of Computer and System Sciences, vol. 63 , pp. 21-41, 2001.

[26] J. Feigenbaum, C. Papadimitriou, S. Shenker, and S. Rahul, "A BGPbased mechanism for lowest-cost routing," in ACM PODC 2002. 